PROYECCIONES

Revista de Matemática Vol. 8/9 Pág. 73-83

$1989 / 90$ - ISSN 0716-0917

Universidad Católica del Norte

Antofagasta - Chile

\title{
IDENTIFICABILIDAD EN ALGUNOS MODELOS EN \\ SERIES DE TIEMPO.
}

\section{Gloria Muñiz S. \\ Jimmy Reyes R.}

\author{
RES U M E N
}

\begin{abstract}
La estructura de un modelo en series de tiempo, en cuanto a su identificacion, constituye un paso pre vio a la estimación de los parámetros que lo caracte rizan.

E'n este articulo se presentan algunos de los métodos clásicos en series de tiempo con una notación no tra dicional, los cuales se descompondrán siempre comó una señal más un ruido. Se entregaran las ideas basicas (definiciones y teoremas) para determinar mas adelante cuando un modelo es identificable utilizando el rango de la matriz de Hankel.
\end{abstract}

\section{INTRODUCCION}

\section{Modelos Arma}

guiente forma:

El problema que nos plantearemos 10 podemos sintetizar de la si

Dado un modelo:

$$
(1+A(L)) Y_{k}=\left(1+C(L) \varepsilon_{k}\right.
$$

que representa la ecuación de un modelo $\operatorname{ARMA}(p, q)$ con $q \leqslant p$, ¿Cómo identificar los parámetros " $p$ " y "q" que caracterizan a este mode10 ? 
1.1. Es importante recordar que los modelos estocásticos (que presentan fenómenos aleatorios que evolucionan en el tiempo de acuerdo a leyes probabilisticas) de clase ARMA en generaT se pueden construir usando como base una extensión de los modelos AR y MA.

La forma generat para un modelo autorregresivo de orden $P[A R(p)]$ estará dada por:

$$
\begin{aligned}
& Y_{k}=S_{k}+\varepsilon_{k} \\
& S_{k}=\alpha(L) Y_{k}
\end{aligned}
$$

en que:

- L es el operador retroceso tal que $L Y_{k}=Y_{k-1}$

$-\alpha(L): \sum_{i=1}^{p} \alpha_{i} L^{i} ; \alpha_{i} \in R$

- $\varepsilon_{k}$ es una secuencia de ruidos blancos no correlacionados.

Esto nos permite indicar que, un $\operatorname{AR}(p)$, tendrá la forma general:

$$
\begin{aligned}
& Y_{k}=\alpha(L) \cdot Y_{k}+\varepsilon_{k} \\
& Y_{k}=\left(\sum_{j=1}^{p} \alpha_{j} L^{i}\right) Y_{k}+\varepsilon_{k}
\end{aligned}
$$

$\therefore \quad$ (1) $Y_{k}=\alpha_{1} Y_{k-1}+\alpha_{2} Y_{k-2}+\ldots+\alpha_{p} Y_{k-p}+\varepsilon_{k}$

con 10 cual podemos concluir que el valor actual del proceso puede expresarse como una suma ponderada de valores pasados más el "ruido" (perturba ción) actua?.

Luego podemos reescribir la ecuación (1) como:

$$
Y_{k}+A(L) Y_{k}=\varepsilon_{k} \Longleftrightarrow(1+A(L)) Y_{k}=\varepsilon_{k}
$$

en que: $1+A(L)=1+\sum_{i=1}^{p} a_{i} L^{i}, \quad$ con $a_{i}=-\alpha_{i} ; \quad \forall_{i}=1,2, \ldots, p$ se 1 lama operador autorregresivo de orden $p$.

1.2. Por otra parte podemos indicar que un $M A(q)$ tendrá la forma:

$$
\begin{aligned}
& Y_{k}=S_{k}+E_{k} \\
& S_{k}=C(L) \varepsilon_{k}
\end{aligned}
$$

en que:

$-C(L)=\sum_{i=1}^{Q} C_{i} L^{i} ; \quad C_{i} \in \ell$ 
análogamente, tendremos entonces que, un $M A(q)$ tendrá la forma general:

$$
\begin{aligned}
& Y_{k}=C(L) \varepsilon_{k}+\varepsilon_{k} \\
& Y_{k}=\left(\sum_{i=1}^{q} C_{i} L^{i}\right) \varepsilon_{k}+\varepsilon_{k}
\end{aligned}
$$

$\therefore \quad$ (2) $\gamma_{k}=c_{1} \varepsilon_{k-1}+c_{2} \varepsilon_{k-2}+\ldots+c_{q} \varepsilon_{k-q}+\varepsilon_{k}$

que podrá escribirse como:

$$
Y_{k}=(C(L)) \varepsilon_{k}+\varepsilon_{k}
$$

en que $C(L)=c_{1} L+c_{2} L^{2}+\ldots+c_{q} L^{q}$

1.3. De tales procesos es posible entonces crear la extensión o modelo mix to 11 amado ARMA $(p, q)$.

$$
\begin{gathered}
Y_{k}=\left[\alpha_{1} Y_{k-1}+\alpha_{2} Y_{k-2}+\ldots+\alpha_{p} Y_{k-p}\right]+ \\
{\left[\varepsilon_{k}+c_{1} \varepsilon_{k-1}+\ldots+{ }_{q} \varepsilon_{k-q}\right]}
\end{gathered}
$$

y convenientemente podrá escribirse como:

$$
(1+A(L)) Y_{k}=(1+C(L)) \varepsilon_{k}
$$

Esto implica entonces, 1a existencia de un parámetro $\theta$ con $(p+q)$ componentes. Donde,

$$
\underline{\theta}^{\prime}=\left(a_{1}, a_{2}, \ldots, a_{p}, c_{1}, c_{2}, \ldots, c_{q}\right)
$$

1.4. Si tenemos la serie de tiempo $\left\{x_{t} \mid t \quad 6 z\right\}$ se define la función de co

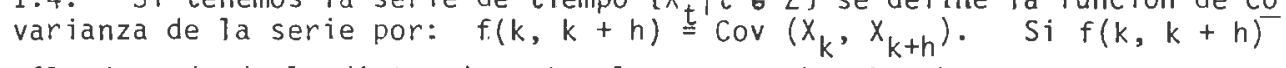
sólo depende de la distancia entre los puntos $k y k+h$, esto es $f(k, k+h)=R_{h}$, entonces $R_{h}$ se denomina función de autocovarianza de la serie.

Así se tendrá que:

$$
R_{h}=\operatorname{Cov}\left(x_{k}, x_{k+h}\right)=\operatorname{Cov}_{\ell}\left(x, x_{\ell+h}\right) \forall k \neq \ell \in Z,
$$

y si la media de la serie es cero entonces $R_{h}=E_{\underline{\theta}}\left[x_{k} X_{k+h}\right]$ 


\section{IDENTIF ICABIL IDAD}

El problema de identificabilidad puede ser enfocado desde dos puntos de vista: i) ¿Qué condiciones son necesarias sobre los parámetros de un modelo para asegurar que éste puede ser simulado con propiedades pres critas? ij) ¿Cuánto pueden revelar los datos acerca del valor de los pá rámetros del modelo?

Para formalizar la identificabilidad entregaremos primeramente la definición de modelo.

\section{Definición 1 .}

Si $Y$ denota los datos de una serie de tiempo. Un modelo $M$ pa-

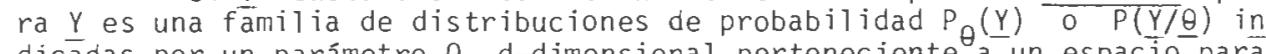
dicādas por un parámetro $\underline{\theta}$ d-dimensional perteneciente $\theta_{\text {a }}$ un espacjo pară metral $R_{\theta} C R^{d}$. Un elemento de $M$; es decir una particular distribución de probabilidades es 11 amiada estructura (o un valor parametral) y la deno taremos por $M_{\theta}(0 \underline{\theta})$.

\section{Definición 2 .}

Dos estructuras $M_{\theta}$ y $M_{\underline{\theta}}^{\prime}, \quad$ (o valores parametrales $\underline{\theta}$ y $\underline{\theta}^{\prime}$ ) son observacionalmente equivalentes si $P_{\underline{\theta}}(\underline{Y})=P_{\underline{\theta}^{\prime}}(\underline{Y})$.

Definición 3 .

Una función $g(\underline{\theta})$ es identificable si y solo si $\left(P_{\underline{\theta}_{1}}(Y)=P_{\underline{\theta}_{2}}(Y)\right.$

$$
\left(P_{\underline{\theta}_{1}}(Y)=P_{\underline{\theta}_{2}}(Y) \Longrightarrow g\left(\underline{\theta}_{1}\right)=g\left(\underline{\theta}_{2}\right)\right. \text {, }
$$

es decir, estructuras observacionalmente equivalentes dan el mismo valor para $g(\underline{\theta})$.

Una aproximación básica para identificabilidad es la Teoría de Funciones Estimables.

Definición 4 .

Una funcion $g(\underline{\theta})$ es estimable si existe una función de los datos $\phi_{g}(y)$ tal que

$$
g(\underline{\theta})=E_{\theta}\left[\phi_{g}(\underline{Y})\right]=\int \phi_{g}(\underline{Y}) P_{\underline{\theta}}(d Y) .
$$

Nota 1. Particularmente, si definimos para algún $i \in Z, h \in Z$.

$$
\phi(Y)=Y_{i} \cdot Y_{i+h} \Longrightarrow R_{h}=E_{\underline{\theta}}\left[Y_{j} Y_{j+h}\right]=g(\underline{\theta})
$$

Luego, $R_{h}$ es estimable. 
Lema 1 : Una función $g(\underline{\theta})$ es identificable si $g(\underline{\theta})$ es estimable.

Demostración

Entonces

$$
\text { Sea } P_{\underline{\theta}_{1}}(y)=P_{\underline{\theta}_{2}}(y)
$$

$$
\begin{aligned}
g\left(\underline{\theta}_{1}\right) & =E_{\underline{\theta}_{1}}\left(\phi_{g}(y)\right)=\int \phi_{g}(y) P_{\underline{\theta}_{1}}(d y) \\
& =\int \phi_{g}(y) P_{\underline{\theta}_{2}}(d y)=g\left(\underline{\theta}_{2}\right)
\end{aligned}
$$

Nota 2 : De acuerdo al modelo: $(1+A(L)) Y_{k}=(1+C(L)) \varepsilon_{k} \Longleftrightarrow$

$$
Y_{k}+\sum_{i=1}^{p} a_{i} Y_{k-i}=\varepsilon_{k}+\sum_{i=1}^{q} c_{i} \varepsilon_{k-i}
$$

Es posible asegurar que:

$$
g(\underline{\theta})=E_{\underline{\theta}}\left[Y_{i} Y_{i+k}\right]=R_{k} \quad k=0, \pm 1, \pm 2, \ldots
$$

es estimable según 1 anterior. Luego podemos asegurar que, ella será iden tificable (por lema 1):

$$
\text { Dado el vector aleatorio: }
$$$$
\underline{Y}=\left[\begin{array}{c}
Y_{1} \\
\vdots \\
Y_{n}
\end{array}\right]
$$

, es posible determinar la matriz de covarianzas:

$$
\operatorname{Cov}[\underline{Y}]=E_{\underline{\theta}}\left[\underline{Y} \underline{Y}^{\prime}\right]=[R|i-j| \underline{(\theta)}], \quad 1 \leqslant i, j \leqslant n
$$

Por 10 tanto, suponiendo distribución normal se tendrá toda la información sobre las $Y_{j}$. (Dado que la función de covarianza y el vector de medias identifican la distribución normal). Así, en series de tiempo las autocovarianzas se toman a menudo como punto de partida para consideraciones de identificabilidad. Retomando la expresión:

$$
\gamma_{k}+a_{1} \gamma_{k-1}+\ldots+a_{p} \gamma_{k-p}=\varepsilon_{k}+c_{1} \varepsilon_{k-1}+\ldots+c_{q} \varepsilon_{k-q}
$$

consideremos $Y_{k-j}$ con $j \geqslant p$.

Si multiplicamos (3) por $Y_{k-j}$ se tendrá:

$$
\begin{aligned}
Y_{k} Y_{k-j}+ & a_{1} Y_{k-1} Y_{k-j}+\ldots+a_{p} Y_{k-p} Y_{k-j}=\varepsilon_{k} Y_{k-j}+\ldots \\
\ldots & +c_{q} \varepsilon_{k-q} Y_{k-j} ; \quad y \text { aplicando esperanza se tendrá: }
\end{aligned}
$$




$$
R_{j}+a_{1} R_{j-1}+\ldots+a_{p} R_{j-p}=E_{\underline{\theta}}\left(E_{k} \cdot Y_{k-j}\right)+\sum_{i=1}^{q} c_{i} E\left[\varepsilon_{k-i} Y_{k-j}\right]
$$
2.7. Denotemos ${ }_{a}^{a}=\left(a_{1}, \ldots ., a_{p}\right)^{\prime}$, y construyamos una matriz $\underline{H}_{p}(\underline{\theta})$
como:

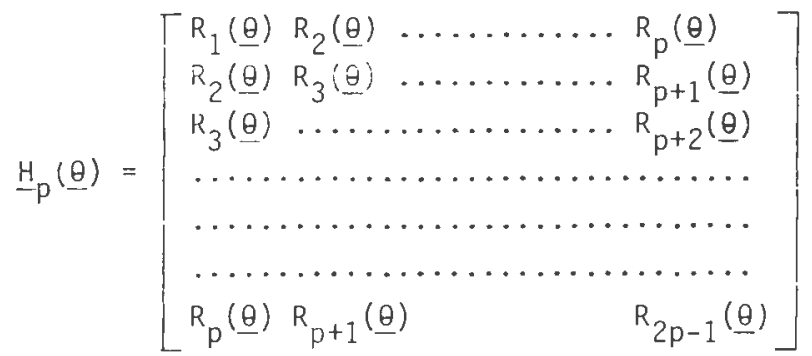

donde $R_{h}(\underline{\theta})=E_{\underline{\theta}}\left[Y_{i} Y_{i+h}\right]=R_{h}$

Esta matriz $\underline{H}_{p}(\underline{\theta})$ se 11 ama matriz de Hankel, y se caracteriza por tener las mismas entradas a 10 largo de las antidiagonales.

Ejemplo

Para el caso de un modelo $\operatorname{ARMA}(2,0)$ es posible construir la ma triz de Hankel teniendo en cuenta 10 siguiente:

En este caso:

$$
\underline{a}_{p}^{\prime}=\left(a_{1}, a_{2}\right)
$$

Además, $\mathrm{R}_{k}(\underline{\theta})=\mathrm{a}_{1} \mathrm{R}_{\mathrm{k}-1}(\underline{\theta})+\mathrm{a}_{2} \mathrm{R}_{\mathrm{k}-2}(\underline{\theta})$

$$
\begin{aligned}
\therefore \quad 1 .-R_{1}(\underline{\theta}) & =a_{1} R_{0}(\underline{\theta})+a_{2} R_{1}(\underline{\theta}) \\
\text { 2. }- & R_{2}(\underline{\theta})=a_{1} R_{1}(\underline{\theta})+a_{2} R_{2}(\underline{\theta})
\end{aligned}
$$

De 1) $\left(1-a_{2}\right) R_{1}(\underline{\theta})=a_{1} R_{0}(\underline{\theta})$

$$
\therefore \quad R_{1}(\theta)=\left(\frac{a_{1}}{1-a_{2}}\right) R_{0}(\underline{\theta})
$$

Con 10 cual

$$
R_{2}(\underline{\theta})=\left(\frac{a_{1}^{2}}{1-a_{2}}+a_{2}\right) R_{0}(\underline{\theta})=R_{0}(\underline{\theta})\left[\frac{a_{1}^{2}+a_{2}-a_{2}^{2}}{1-a_{2}}\right]
$$

Como:

$$
R_{3}(\underline{\theta})=a_{1} R_{2}(\underline{\theta})+a_{2} R_{1}(\underline{\theta})
$$




$$
\therefore \quad R_{3}(\underline{\theta})=R_{0}(\underline{\theta})\left[\frac{a_{1}^{3}}{1-a_{2}}+a_{2} a_{1}+\frac{a_{1} a_{2}}{1-a_{2}}\right]
$$

Que podemos escribir como:

$R_{3}(\underline{\theta})=R_{0}\left(\theta_{-}\right)\left[\frac{a_{1}^{3}+a_{1} a_{2}-a_{2}^{2} a_{1}+a_{1} a_{2}}{1-a_{2}}\right]=R_{0}(\underline{\theta})\left[\frac{a_{1}^{3}+2 a_{1} a_{2}-a_{2}^{2} a_{1}}{1-a_{2}}\right]$

Con 10 cual la matriz de Hankel $H_{p}(\underline{\theta})$ tendrá la forma:

$$
\underline{H}_{p}(\underline{\theta})=\frac{R_{0}(\underline{\theta})}{1-a_{2}}\left[\begin{array}{ll}
a_{1} & a_{1}^{2}+a_{2}-a_{2}^{2} \\
a_{1}^{2}+a_{2}-a_{2}^{2} & a_{1}^{3}+2 a_{2} a_{1}-a_{2}^{2} a_{1}
\end{array}\right]
$$
2.8. Suponiendo " $p$ " desconocido, podemos obtener $\underline{a}_{p}$ al resolver la ecua-
ción:

(6)

$$
H_{p}(\underline{\theta}) \cdot \underline{a}_{p}=r_{p}(\underline{\theta})
$$

donde :

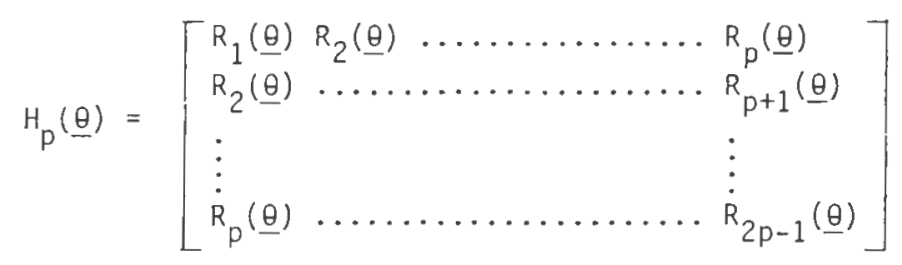

es la matriz de Hankel y

$$
r_{p}(\underline{\theta})=\left[\begin{array}{l}
R_{p+1}(\theta) \\
\vdots \\
\dot{R}_{2 p}(\theta)
\end{array}\right]
$$

\subsection{Teorema 1:}

$$
\begin{aligned}
& \text { En el modelo ARMA }(p, q) ; q \leqslant p \\
& (1+A(L)) Y_{k}=(1+C(L)) \varepsilon_{k}, \text { donde } \\
& A(L)=\sum_{i=1}^{p} a_{i} L^{i} \text { y } C(L)=\sum_{i=1}^{q} c_{i} L^{i}
\end{aligned}
$$

${ }_{-p}$ es identificable si y solamente si el rango de $\underline{H}_{p}(\underline{\theta})$ es $p$. 


\section{Demostración}

$$
\text { Sea } \underline{\theta}^{\prime}=\left(\underline{a}_{p}^{\prime}, \underline{c}_{p}^{\prime}\right)=\left(a_{1}, \ldots, a_{p}, c_{1} \ldots, c_{q}\right) \text { y } g(\underline{\theta})=\underline{a}_{p} .
$$

Entonces $a_{p}$ es identificable $\Longrightarrow$

$$
\left(P_{\theta_{1}}(\underline{y})=P_{\underline{\theta}_{2}}(\underline{y}) \Longrightarrow \underline{a}_{p_{1}}={\stackrel{a}{p_{2}}}_{2}\right) \quad \begin{aligned}
& \text { (Por definición de identificabili- } \\
& \text { dad). }
\end{aligned}
$$

$$
\text { Supongamos: }{ }_{\underline{e}}(\underline{Y})=P_{\underline{\theta}_{2}}(\underline{Y}) \text {. }
$$

Ya que las $R_{k}(\underline{\theta})$ son determinadas por $P_{\underline{\theta}}(\underline{Y})$, entonces los $\underline{a}_{-p}$ son identi-
ficabies

$$
R_{k}\left(\underline{\theta}_{1}\right)=R_{k}\left(\underline{\theta}_{2}\right) \quad \forall_{k} \Longrightarrow \underline{a}_{p_{1}}=\underline{a}_{p_{2}}
$$

$1 ! 2 g \circ$ por ser $R_{k}(\underline{\theta})_{1}=R_{k}(\theta)_{2} \quad \forall_{k} \Longrightarrow$

$$
r_{p}\left(\underline{\theta}_{1}\right)=\left[\begin{array}{c}
R_{p+1}\left(\underline{\theta}_{1}\right) \\
\vdots \\
\dot{R}_{2 p}\left(\underline{\theta}_{1}\right)
\end{array}\right]=\left[\begin{array}{c}
R_{p+1}\left(\underline{\theta}_{2}\right) \\
\vdots \\
\dot{R}_{2 p}\left(\underline{\theta}_{2}\right)
\end{array}\right]=r_{p}\left(\underline{\theta}_{2}\right)
$$

Luego, de las ecuaciones:

$$
\begin{aligned}
& \stackrel{H}{p}_{p}\left(\underline{\theta}_{1}\right) \cdot{\stackrel{a}{p_{1}}}_{1} \underline{-r}_{p}\left(\underline{\theta}_{1}\right) \\
& { }_{-p}^{H}\left(\theta_{2}\right) \cdot \underline{a}_{p_{2}}={ }_{-p}^{r}\left(\underline{\theta}_{2}\right)
\end{aligned} \Longrightarrow \underline{H}_{p}\left(\underline{\theta}_{1}\right)=\underline{H}_{p}\left(\underline{\theta}_{2}\right)
$$

Haciendo:

$$
\begin{gathered}
\underline{r}_{p}=\underline{r}_{p}\left(\underline{\theta}_{1}\right)=\underline{r}_{p}\left(\underline{\theta}_{2}\right) \\
H_{p}=\underline{H}_{p}\left(\underline{\theta}_{-}\right)={ }_{p}\left(\underline{\theta}_{2}\right), \text { entonces: }
\end{gathered}
$$

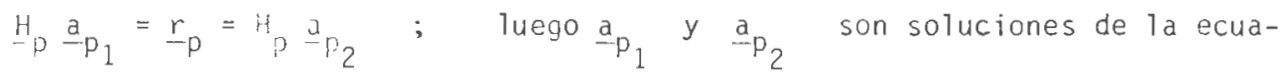
ción $\underline{H}_{p} a_{p}=\underline{r}_{p} \quad y$ šsta tiene solución ünica ssi rango $\underline{H}_{p}=p$.

$$
\therefore \quad{ }_{a}^{a} p_{1}={ }_{-}^{a} p_{2} \Longleftrightarrow \quad \text { Rango } \underline{H}_{p}=p
$$

luego suponiendo, 


$$
P_{\underline{\theta}_{1}}(\underline{y})=P_{\underline{\theta}_{2}}(\underline{y}) ; \quad \underline{a}_{p} \text { identificable } \Longleftrightarrow \underline{a}_{p_{1}}=\underline{a}_{p_{2}} \Leftrightarrow
$$

Rango $\underline{H}_{p}=p$

Nota 3. Un método de análisis numérico estandar para investigar rangos es eT 1 lamado Descomposición en VaTores Singulares (S.V.D.), anal izado en strang (1974) "Algebra Lineal y sus aplicaciones".

Fero, desde un puito de vista estadistico es más natural reducir la escala $\underline{H}_{p}=\underline{H}_{p}(\underline{\theta})$ usando

$$
\ddot{H}_{p}=R_{p}^{-1 / 2} \underline{H}_{p} \underline{R}_{p}^{-1 / 2} \quad \text { donde } \underline{R}_{p}^{1 / 2} \text { es la raíz cua }
$$

drada de $\underline{R}_{p}=\left[R_{|i-j|}\right] \quad 1 \leqslant i, \quad j \leqslant p$

En la práctica, se reemplaza $R_{k}$ por :

$$
\hat{R}_{k}=\frac{1}{n}\left[\sum_{i=1}^{n-k} Y_{i} \cdot Y_{i+k}\right] \quad \text { que representan las covarian- }
$$

zas muestrales; estos valores son conocidos ya que $\mathbf{n}$ representa el nümero de observaciones, y los valores de $Y_{i}, Y_{i+k}$ corresponden a los valores de las observaciones; y realizar un S.V.D. de $\tilde{H}$, donde el valor de $v$ corresponderá a una cota superior (mayor valor) 'v'del orden del proceso ARMA.

$$
\begin{gathered}
\text { Al no conocer todavia } \underline{H}_{v} \text { ésta se considerará como: } \\
\underline{H}_{v}=E\left[\underline{Y}_{0}^{+} \underline{Y}_{0}^{-1}\right]
\end{gathered}
$$

en que:

$$
\begin{aligned}
& \underline{Y}_{i}^{+}=\left(Y_{i}, Y_{i+1}, \ldots ., Y_{i+(v-1)}\right)^{\prime} \\
& \underline{Y}_{i}^{-}=\left(Y_{i-1}, Y_{i-2}, \ldots ., Y_{i-v}\right)^{\prime}
\end{aligned}
$$

con lo cual,

$$
\begin{aligned}
& \underline{Y}_{0}^{+}=\left(Y_{0}, Y_{1}, \ldots, Y_{V-1}\right)^{\prime} \\
& \underline{Y}_{0}^{-}=\left(Y_{-1}, Y_{-2}, \ldots, Y_{-V}\right)^{\prime}
\end{aligned}
$$

En la práctica, se tiene entonces que:

$$
\underline{H}_{v}=\underline{R}_{v}^{1 / 2} E\left[\underline{Y}_{-0}^{+} \underline{Y}_{0}^{-1}\right] \cdot \underline{R}_{v}^{-1 / 2}
$$


Se tiene entonces que $\underline{\tilde{H}}_{v}$ es una matriz de orden $v$ y además que $\underline{H}_{v}=E\left[\underline{Y}_{0}^{+} \underline{Y}_{-0}^{-}\right]$.

Nota 4. De acuerdo al teorema 1 podemos definir el orden de un modelo ARMA univariado, como el minimo "p" tal que

$$
\text { Rango }\left(\underline{H}_{p}\right)=\operatorname{Rango}\left(\underline{H}_{p+i}\right), \quad i \geqslant 1
$$

Existe también otra forma para determinar el orden que es útil para series de tiempo multivariadas.

Denotemos:

$$
\left.Y_{k+j}\right|_{k-1}=E\left(Y_{k+j} \mid \varepsilon_{k-1}, \cdots, \varepsilon_{1}\right)
$$

Fara el modelo ARMA dado al comienzo podemos encontrar que

$$
\left.Y_{k+j+p}\right|_{k-1}+\left.a_{1} Y_{k+j+p-1}\right|_{k-1}+\ldots+\left.a_{p} Y_{k+j}\right|_{k-1}=0, \quad j \geqslant 0
$$

pues los ruidos no están correlacionados para $j \geqslant 0$. De esta forma pode mos decir:

El orden es el menor indice p tal que

$Y_{k+p \mid k-1}$ es 1 inealmente dependiente sobre sus predecesores

$$
\left.Y_{k+i}\right|_{k-1} \quad 0 \leqslant i \leqslant p-1
$$

Esto se relaciona con la definición indicada en la Nota 4 , de la siguiente forma:

$$
\begin{aligned}
& \text { Observemos que: } \\
& \underline{H}_{v}=E\left(\underline{Y}_{0}^{+} \underline{Y}_{0}^{-1}\right)=E\left[\underline{Y}_{k}^{+} \underline{Y}_{k}^{-1}\right]=E \\
& {\left[\begin{array}{l}
Y_{k} \\
\vdots \\
Y_{k+v-1}
\end{array}\right]} \\
& \left(Y_{k-1}, \ldots, Y_{k-v}\right) \\
& =E\left(\left[\begin{array}{l}
\left.Y_{k}\right|_{k-1} \\
\vdots \\
\left.Y_{k+v-1}\right|_{k-1}
\end{array}\right]\left(Y_{k-1} \ldots, Y_{k-v}\right)\right)
\end{aligned}
$$

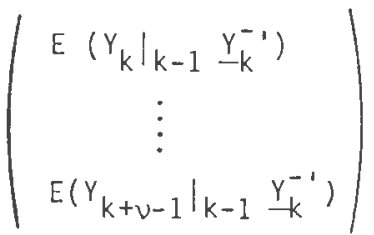


De esta forma $H_{V}$ tiene rango $\mathrm{p}$ si y solamente si tiene $\mathrm{p}$ columnas linealmente independientes to que se cumple claramente si 5.1 . se cum ple.

\section{REFERENCIAS}

[1] KAILATH, T. "Linear Systems". Prentice Hall, U.S.A., 1980.

[2] SOLO, V. "Topics in advanced time Series Analysis". Harvard Uni versity, Department of Statistics. Lectures presented at Primera Escuela de Invierno de Probabilidad y Estadistica. Faculty of Mathematics, Department of Statistics, Pontificia Universidad Católica de Chile. July 1982.

I. Gloria Muñiz S.* Jimmy Reyes R.

* Departamento de Matemáticas. Facultad de ciencias. Universidad Católica del Norte. Casilla 1280.

* Departamento de Matemáticas. Facuitad de Ciencias Básicas.

universidad de Antofagasta. Casilia 170 .

Antofagasta - CHILE 
PROYECCIONES es una publicación oficial de la Universidad Católica del Norte. Fue fundada en el año 1982 y autorizada por Resolución Exenta del Ministerio del Interior No 259 de 1983. Actualmente depende del Departamento de Matemáticas de la Facultad de Ciencias de la Universidad Católica del Norte.

$\begin{array}{lll}\text { Director Responsable } & : & \text { Ricardo Soto Montero } \\ \text { Editor Ejecutivo } & : \text { Julio Peña Rodríguez } \\ \text { Comité Editorial } & : \text { Miguel Blázquez Jiménez - Universidad Técnica Federico } \\ & \text { Santa María } \\ & \text { Ramón Correa Soto - Universidad Católica del Norte } \\ & \text { Eduardo Muñoz Morales - Universidad Católica del Norte } \\ & \text { Samuel Navarro - Universidad de Santiago de Chile } \\ & \text { Arnoldo Prado Campos - Universidad Católica del Norte } \\ & \text { Rolando Rebolledo - Pontificia Universidad Católica de Chile } \\ & \text { Héctor Rojo Jeraldo - Universidad de Antofagasta } \\ & \text { Oscar Rojo Jeraldo - Universidad Católica del Norte } \\ & \text { Ricardo Soto Montero - Universidad Católica del Norte } \\ & \text { Nicolás Yus - Universidad de Chile }\end{array}$

Dirección Casa Central : Avenida Angamos 0610, Antofagasta, Chile

Dirección Postal : Casilla 1280, Antofagasta, Chile

Publicación Periódica : ISSN $0716-0917$ 